\title{
ANISOTROPY STUDY OF THE FEBRUARY 4TH 2008 SWARM IN NW PELOPONNESUS (GREECE)
}

\author{
Kaviris G. ${ }^{1}$, Papadimitriou P. ${ }^{1}$, and Makropoulos K. ${ }^{1}$ \\ ${ }^{1}$ Department of Geophysics, University of Athens, 15784 Athens, Greece, \\ gkaviris@geol.uoa.gr,ppapadim@geol.uoa.gr,kmacrop@geol.uoa.gr
}

\begin{abstract}
The Gulf of Corinth, located in central Greece, is characterized by normal faulting and by high seismicity since the antiquity. On 4 February 2008 a seismic swarm burst in NW Peloponnesus characterized by the occurrence of two moderate-size earthquakes of moment magnitudes 4.7 and 4.5, respectively. Analysis of the data revealed the existence of shear-wave splitting. The events that were chosen for the anisotropy study fit the selection criteria, having clear and impulsive $S$ wave arrival phases on the horizontal components. In addition, the amplitude of the $S$ wave phase on the vertical component was smaller than on the horizontal ones. The representations that were used to determine the polarization direction of the fast split shear wave, the time delay between the two split shear waves and the polarization direction of the source were the polarigram and the hodogram. The uniform fast shear wave polarizations, irrespective of the azimuth of each event, are consistent with the general NNE-SSW direction of extension in the Gulf and, therefore, in agreement with the extensive dilatancy anisotropy (EDA) model. Finally, a decrease of the time delay values was observed after the occurrence of the first moderate earthquake, implying changes of the medium's properties.
\end{abstract}

Key words: Anisotropy, shear wave splitting, temporal variation, stress field, EDA model, Gulf of Corinth, NW Peloponnesus.

\section{Introduction}

The Gulf of Corinth, located in Central Greece, is one of the faster expanding and most seismically active continental rifts around the world and is characterized by normal faulting in an approximate E-W direction. Several strong historical earthquakes have destroyed cities in the Gulf, such as Heliki in 373 BC. Since 1911, the instrumental seismicity in the Gulf of Corinth is also intense (Makropoulos and Burton, 1981; Ambraseys \& Jackson 1990) and includes several large events with $\mathrm{M}>6$ from 1928 (Corinth, Ms 6.3) until 1995 (Aigion, Ms 6.2). In NW Peloponnesus, in the area between the Corinth Rift and Zakynthos island, there is a transition zone characterised by dextral strike-slip faulting.

Four months before the occurrence of the $\mathrm{Mw}=6.4$ Andravida earthquake (Ganas et al., 2009), on 4 February 2008, which could influence the physical properties of the medium in the broader area, a seismic swarm, characterized by two moderate-size earthquakes $\sim \mathrm{M} 4.6$, burst about $40 \mathrm{~km}$ NE of the epicenter of this event. A known normal fault in the vicinity of the epicentral area is the Chalandritsa fault (Ch. F. in Fig. 1), with an approximate E-W strike. It is interest- 


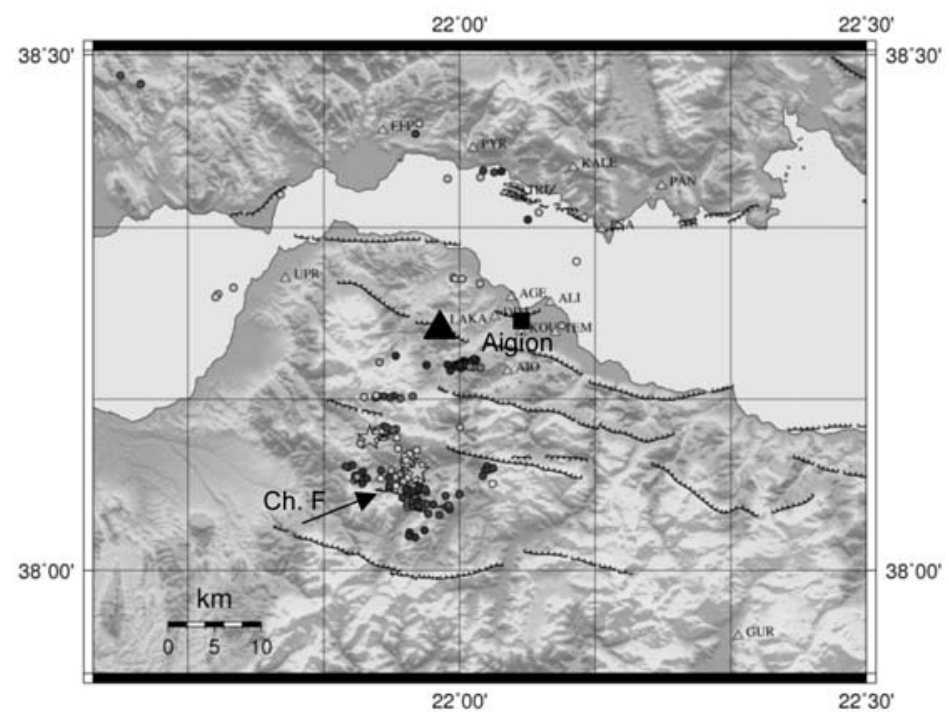

Fig. 1: Location of events of the February $4^{\text {th }} 2008$ swarm in NW Peloponnesus. The major shocks are depicted as stars. The location of LAKA station is also indicated with a triangle. Ch. F. is the Chalandritsa Fault.

ing that the calculated focal mechanisms of the two main events of this swarm reveal dominant strike-slip (Kapetanidis et al., 2008). This is not in agreement with the well-known normal faults of the area which have a dominant E-W strike, thus it is an interesting case of transition between normal and strike-slip faults.

Several anisotropy studies have been performed in Greece. The shear-wave splitting phenomenon was observed in Milos island (Booth et al., 1989), Kalamata (Bouin, 1994), Almyros (Karnassopoulou, 1996), Gulf of Corinth (Bouin et al., 1996; Papadimitriou et al., 1999; Kaviris et al., 2008) and Attica (Papadimitriou et al., 2000), leading to conclusions for the local stress-field.

\section{LAKA Station - Method Used}

LAKA station (Fig. 1) is located on the south coast of the Gulf of Corinth, west of the city of Aigion. This station belongs to the ATHENET network, which is the permanent network of the Seismological Laboratory of the University of Athens and part of the Hellenic Unified Seismological Network. The relatively small epicentral distance of this station from the February 4th 2008 seismic swarm permits the detection of many microearthquakes (Fig. 1).

The analysis of earthquakes of the 4 February 2008 sequence in NW Peloponnesus that were recorded by the LAKA station revealed the existence of shear-wave splitting, which is related to the existence of anisotropic medium. It is worth noticing that the observation of stress aligned seismic shear-wave splitting in almost all in situ rocks, in various sedimentary and crystalline geological regimes, below some critical depth, usually between about $500 \mathrm{~m}$ and $1 \mathrm{~km}$ is nearly universal (Kaneshima, 1990; Crampin and Lovell, 1991; Coutant, 1996; Gamar and Bernard, 1997; Papadimitriou et al., 1999; Crampin and Chastin, 2001; Hao et al., 2008). Shear-waves split into two approximately orthogonal fixed-polarizations with different velocities, which is characteristic of propagation in media with some form of elastic anisotropy. Shear wave splitting can easily be recognized into the polarigram or the three-component particle motion of shear-wave 


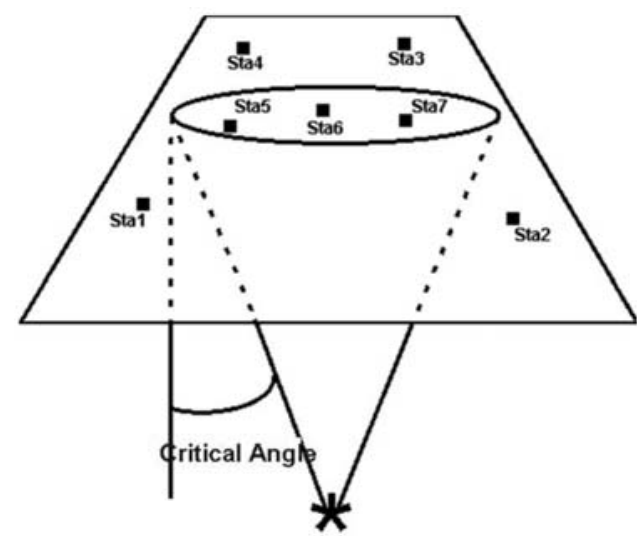

Fig. 2: All events selected for the anisotropy study are located within the shear-wave window, represented by the circle. The critical angle is equal to $45^{\circ}$.

arrivals and is the key diagnostic phenomenon for analyzing seismic anisotropy (Crampin, 2003).

All the events that were selected for the anisotropy study are located close to the LAKA station, within the shear-wave window (Fig. 2), having incident angles smaller than the critical. In addition, they have clear and impulsive $S$ wave arrival phases on the horizontal components, while the amplitude of the $\mathrm{S}$ wave phase on the vertical component is smaller than on the horizontal ones. When the above conditions are true, scattered and converted phases (e.g. SP) are rejected (Booth and Crampin, 1985). A visual inspection and a plot of the particle motion in the three planes of projection were used to select the events that match the criteria mentioned above. The representations used in the present study in order to determine the splitting parameters, which are the polarization direction of the $S_{\text {fast }}$ wave, the time delay between the two split shear waves and the source polarization direction, are the polarigram (Bernard and Zollo, 1989) and the hodogram.

An example of an event that is located close to the LAKA station and fulfils the selection criteria is presented in Figure 3A. A band-pass filter in the frequency range 0.5-25 Hz was used. The event occurred on 3/2/2008 01:02 GMT, with an azimuth equal to $206^{\circ}$ and an angle of incidence equal to $38^{\circ}$, within the shear-wave window. Both the polarigram and the hodogram of the N-E plane are presented, where shear-wave splitting is evident. The angle between the north and the fast axis (S1) is the polarization direction, which is equal to $\mathrm{N} 103^{\circ}$.

Then, the seismograms are rotated in the fast (S1) and slow (S2) direction and the obtained polarigram and hodogram are presented in Figure 3B. In this figure, the obtained polarization vector is oriented almost parallel to the fast component. The measured time delay is equal to $0.070 \mathrm{~s}$, represents the magnitude of the anisotropy and is removed in order to obtain the polarization direction of the source.

To measure the polarization direction of the source, the fast component is temporally moved towards the slow one for an interval of time equal to the time delay $(0.070 \mathrm{sec})$ and the obtained waveforms (recorded and filtered) are presented in Figure 3C. The obtained polarization angle is $57^{\circ}$ from the fast axis $\left(\mathrm{F} 57^{\circ}\right)$. The polarization direction of the source is the sum of this angle and of the polarization direction $\left(\mathrm{N}_{103^{\circ}}\right)$ and is equal to $\mathrm{N} 160^{\circ}$. Following, in Figure $3 \mathrm{D}$, the horizontal components are rerotated to their initial directions (E-W, N-S, angle of rotation $\left.103^{\circ}\right)$. The obtained waveforms are theoretically those that would be recorded in the case that the medium between the hypocenter and the station (LAKA) was not anisotropic. The polarization direction of the source is directly measured and is found equal to $\mathrm{N} 160^{\circ}$. 

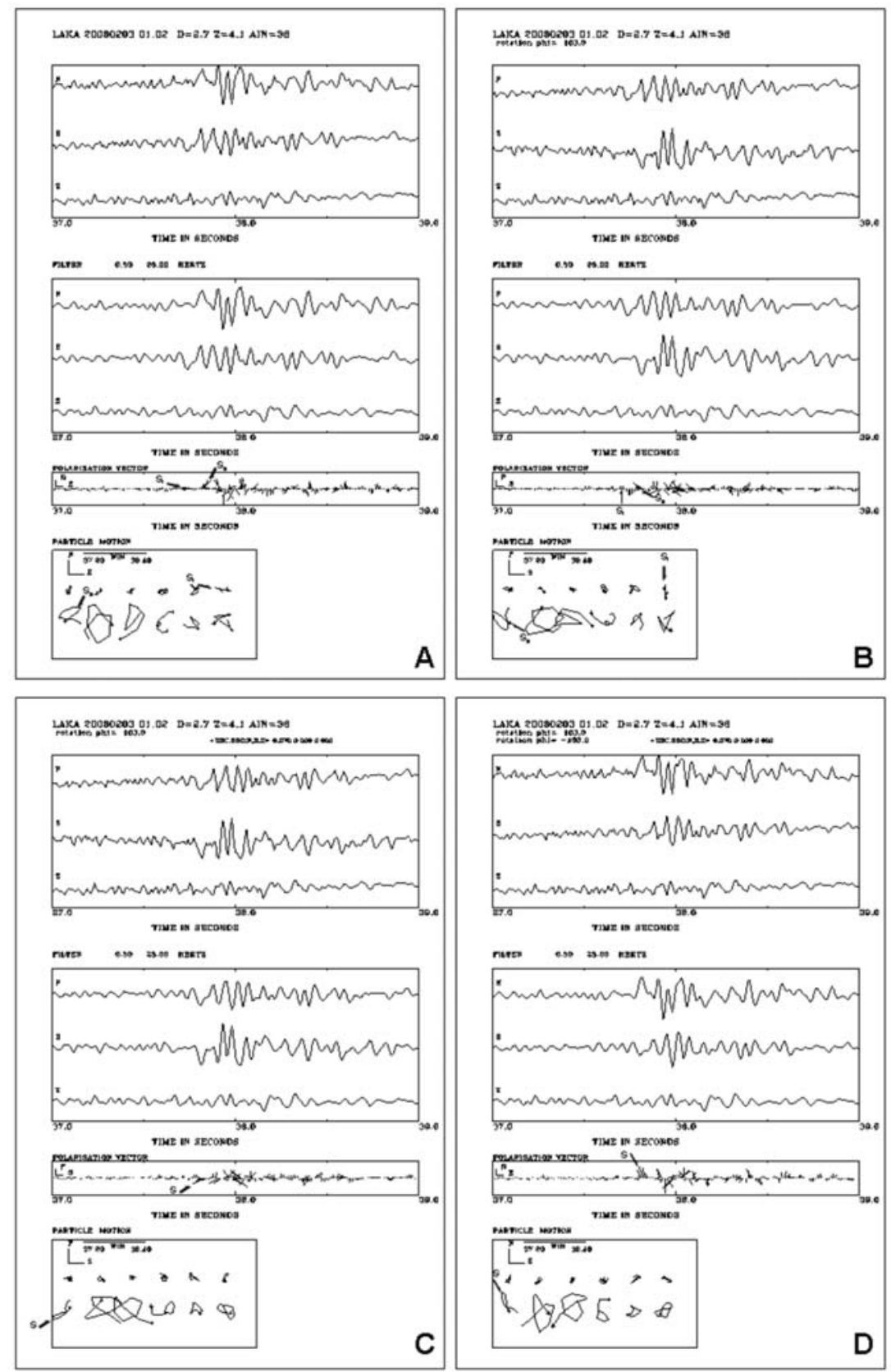

Fig. 3: (A) Original traces of an earthquake recorded at LAKA station, filtered traces, polarigram and hodogram in the N-E plane. (B) Traces rotated parallel and orthogonal to the polarization direction of the fast shear wave, filtered waveforms of the rotated traces, polarization vector and hodogram in the fast-slow plane where the time delay is measured. (C) Traces rotated parallel and orthogonal to the polarization direction of the fast shear wave after the correction of the time delay, filtered waveforms, polarigram and hodogram. (D) Traces re-rotated to the N-S and E-W directions, filtered waveforms, polarigram and hodogram from which the polarization of the source is estimated. 


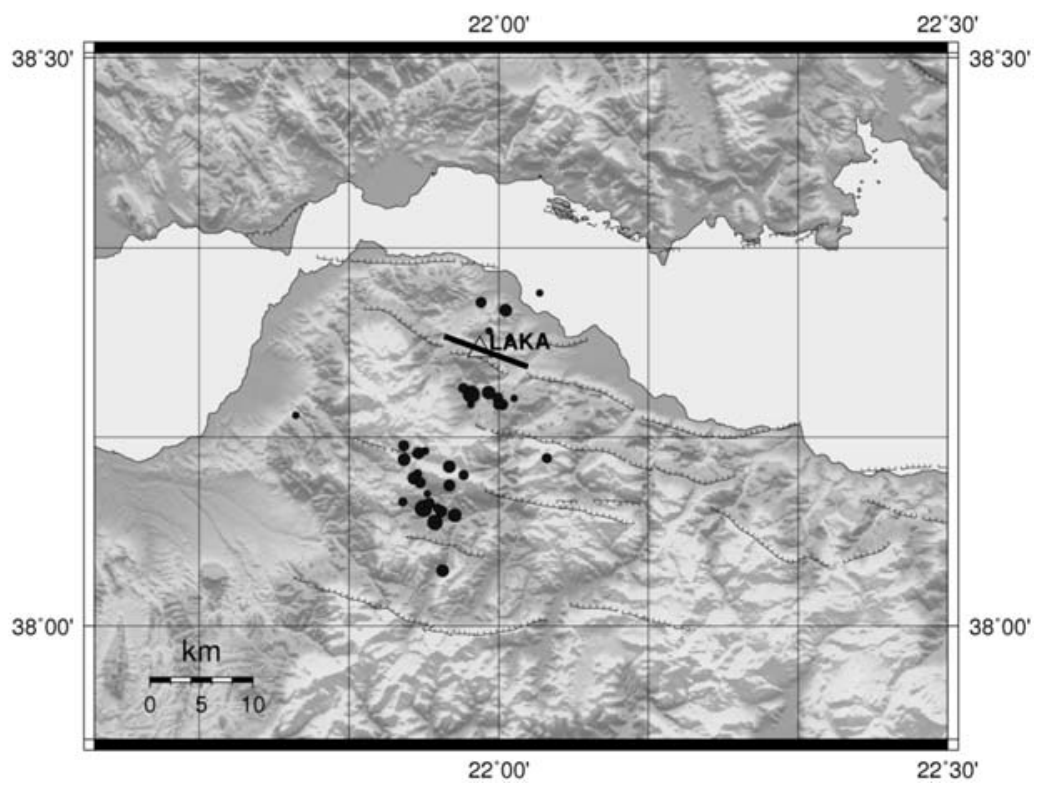

Fig. 4: Selected events for the anisotropy study at LAKA station and mean polarization direction.
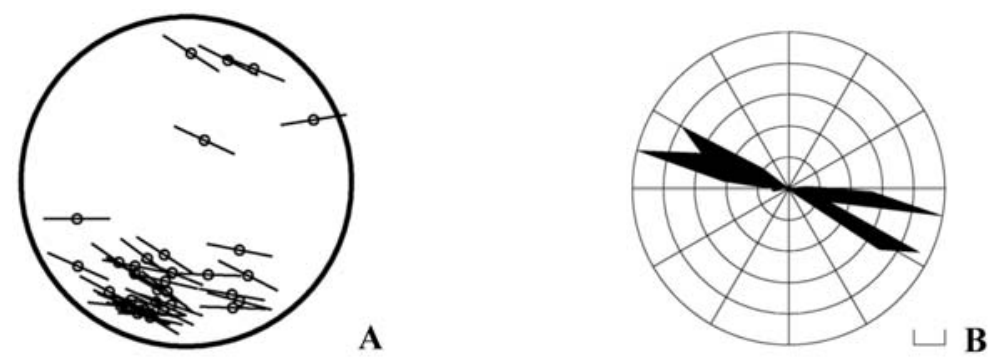

Fig. 5: Present Study: (A) Polar equal-area projections on the upper hemisphere of the fast shear wave polarizations at LAKA station. The circle represents an angle of incidence equal to $45^{\circ}$. (B) Rose diagram of the fast shear wave polarization directions at LAKA station.

\section{Results of the Anisotropy Study}

During the analysis of the events of the 4 February 2008 sequence in NW Peloponnesus that were recorded by the LAKA station, 36 were selected for the anisotropy study (Fig. 4). The angles of incidence of the selected events vary between $14^{\circ}$ and $44^{\circ}$. The back azimuth range of the selected epicenters is quite satisfactory, varying between $2^{\circ}$ and $318^{\circ}$ with two gaps, the first between $64^{\circ}$ and $142^{\circ}$ and the second between $251^{\circ}$ and $318^{\circ}$. Furthermore, the back-azimuths of the $83 \%$ of the selected events vary between $142^{\circ}$ and $232^{\circ}$.

The polarization directions of the fast shear wave are presented on equal-area projections of the upper hemisphere (Fig. 5A). The outer circle defines the shear wave window and represents an angle of incidence equal to $45^{\circ}$. The length of the bars is proportional to the time delay between the fast and slow shear waves. The values of the time delays at LAKA station vary be- 

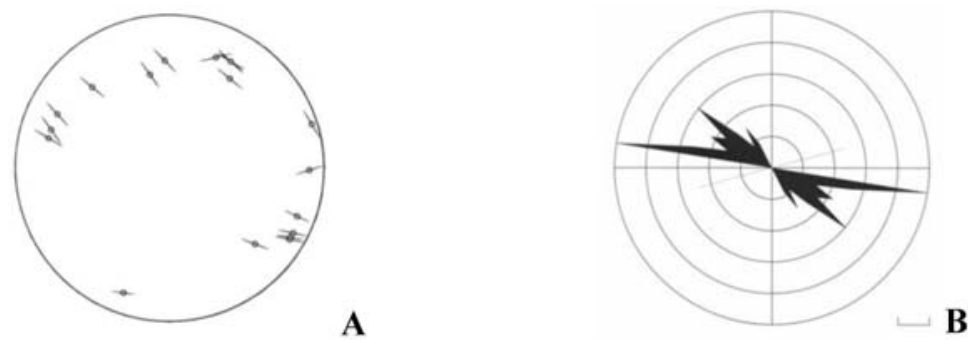

Fig. 6: (A) Polar equal-area projections on the upper hemisphere of the fast shear wave polarizations at LAKA station from Kaviris et al., 2008. (B) Rose diagram of the fast shear wave polarization directions at LAKA station from Kaviris et al., 2008.

tween $0.020 \mathrm{~s}$ and $0.090 \mathrm{~s}$, while the $\mathrm{S}_{\text {fast }}$ polarization directions of the fast shear wave vary between $\mathrm{N} 81^{\circ}$ and $\mathrm{N} 129^{\circ}$. The coherence of the fast shear wave polarizations at LAKA station, irrespective of the azimuth of each event, is consistent with shear-wave splitting due to the seismic wave propagation through an anisotropic medium.

The rose diagram of the $S_{\text {fast }}$ polarization directions for all the selected events at LAKA station is presented in Fig. 5B. Two main $\mathrm{S}_{\text {fast }}$ polarization directions of about $\mathrm{N} 100^{\circ}$ and $\mathrm{N} 120^{\circ}$ are observed, with a mean value equal to $\mathrm{N} 110^{\circ} \pm 2^{\circ}$, as presented in Fig. 4.

It is worth noticing that a previous anisotropy study was also performed for the LAKA station using 19 events recorded during the year 2000 (Kaviris et al., 2008). The values of the time delays for these events varied between $0.024 \mathrm{sec}$ and $0.104 \mathrm{sec}$, while the polarization directions of the fast shear wave between $\mathrm{N} 72^{\circ}$ and $\mathrm{N} 150^{\circ}$ (Fig. 6A). The main $\mathrm{S}_{\text {fast }}$ polarization direction was $\mathrm{N} 123^{\circ}$, while a secondary one equal to $\mathrm{N} 100^{\circ}$ was also observed (Fig. 6B). It is obvious that in both cases similar main polarization directions are obtained, with higher variation in the previous study.

\section{Temporal Variation of time delay}

One of the splitting parameters measured in the present study is the time delay between the two split shear waves. Time delays are sensitive to small changes in microcrack geometry, since changes in shear wave splitting monitor the small-scale stress-induced deformation of microcracks throughout the rock mass before a level of microcracking known as fracture criticality is reached when rocks are expected to fracture (Crampin and Chastin, 2003). Temporal variations in shear-wave time-delays have been observed before several earthquakes with magnitudes M 1.7 to M 7.7 (Crampin and Peacock, 2008). These observations have been performed in different regions, worldwide, such as Iceland (Crampin et al., 1999), China (Gao et al., 1998), U.S.A. (Liu et al., 1997) and Taiwan (Crampin and Gao, 2005). Whenever there is adequate data, these characteristic patterns of temporal variation are seen before all larger earthquakes and earthquakes can be stress-forecasted (Crampin, 1999). A necessary condition for fracturing is that cracks are so closely-spaced that shear-strength is lost and rocks fracture whenever there is any disturbance (Gao and Crampin, 2008).

As it is already mentioned in the previous section, the obtained values of the time delays at LAKA station vary between $0.020 \mathrm{sec}$ and $0.090 \mathrm{sec}$. The first major event of February 4 th 2008 


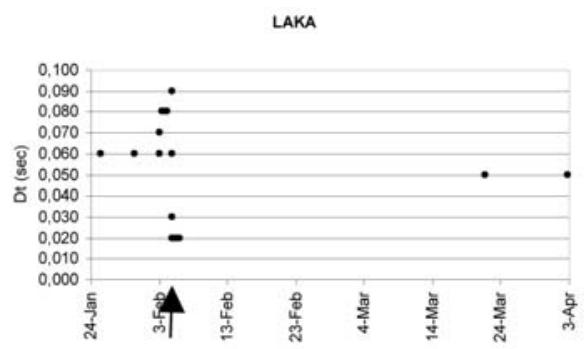

A

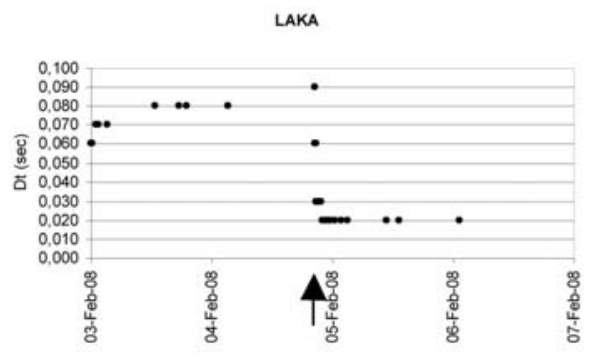

B

Fig. 7: Shear-wave splitting time-delays at LAKA station for the period: (A) 25 January to 2 April 2008 and (B) 3 to 6 February 2008. The arrows indicate the time of the first major event.

occurred at 20:25GMT. It is important to notice that the time delays before the occurrence of this shock vary between 0.060 and $0.090 \mathrm{sec}$, while afterwards between 0.020 and $0.060 \mathrm{sec}$ (Fig. 7A, B). This decrease clearly indicates a change of the medium's properties after the occurrence of the first major event. Various anisotropy studies are performed in Greece, but this is the first time that temporal variation of the time delay is observed before the occurrence of an earthquake.

\section{Conclusions}

On 4 February 2008, two moderate earthquakes $\sim$ M4.6 triggered a seismic swarm in NW Peloponnesus. Analysis of the data revealed the existence of an anisotropic upper crust around LAKA station and an anisotropy study was performed. Using the appropriate selection criteria, a dataset comprising of 36 events was obtained. Scattered and converted phases that could lead to false identification of the split shear waves were identified and rejected.

Almost linear polarization was observed, with a mean direction equal to $\mathrm{N} 110^{\circ}$. The uniformity of the fast shear wave polarizations, irrespective of the azimuth of each event, is consistent with what is expected for shear-wave splitting due to propagation through an anisotropic medium. These observations are consistent with the general NNE-SSW direction of extension in the Gulf of Corinth and, therefore, in agreement with the extensive dilatancy anisotropy (EDA) model. Finally, it is worth mentioning that an important reduction of time delay values was observed immediately after the occurrence of the first major event, implying changes of the medium's properties.

\section{References}

Ambraseys, N. N. and Jackson, J. A., 1990. Seismicity and associated strain in central Greece between 1890 and 1988. Geophys. Jour. Inter., 101, 663-708.

Bernard, P. and Zollo, A., 1989. Inversion of near-source S polarization for parameters of doublecouple point like sources. Bull. Seism. Soc. Am., 79, 1779-1809.

Booth, D. C. and Crampin, S., 1985. Shear wave polarizations on a curved wavefront at an isotropic free surface. Geophys. J. R. Astron. Soc., 83, 31-45.

Booth, D. C., Scjleper, S., Crampin, S., Ochmann, N. and Wohlenberg, J., 1989. Shear-wave splitting observations on Milos, Greece. Geothermics, 18, 597-610. 
Bouin, M.-P., 1994. Analyse de la polarisation des ondes S en source proche: Rupture et Structure. Phd Thesis, Universite Paris 7, Paris.

Bouin, M.P., Tellez, J. and Bernard, P., 1996. Seismic anisotropy around the Gulf of Corinth, Greece, deduced from three-component seismograms of local earthquakes and its relationship with crustal strain. J. Geophys. Res., 101, 5797-5811.

Coutant, O., 1996. Observation of shallow anisotropy on local earthquake records at the Garner Valley, Southern California, downhole array. Bull. Seism. Soc. Am., 86, 477-488.

Crampin, S., 2003. The New Geophysics: shear-wave splitting provides a window into the crack-critical rock mass. The Leading Edge, 22, 536-549.

Crampin, S. and Lovell, H. L., 1991. A decade of shear-wave splitting in the Earth's crust: What does it mean? What can we make of it? and What should we do next? Geophys. J. Int., 107, 387-407.

Crampin, S. and Chastin, S., 2001. Shear-wave splitting in a critical crust: II - compliant, calculable, controllable fluid-rock interactions, in Anisotropy 2000: Fractures, converted waves, and case studies, Proc. 9th Int. Workshop on Seismic Anisotropy, Camp Allen, 2000, eds. Ikelle, L. T. \& Gangi, T., SEG Open File Publication No. 6, 21-48.

Crampin, S. and Chastin, S., 2003. A review of shear-wave splitting in the crack-critical crust. Geophys. J. Int., 155, 221-240.

Crampin, S. and Gao, Y., 2005. Comment on "Systematic analysis of shear-wave splitting in the aftershock zone of the 1999 Chi-Chi, Taiwan, earthquake: shallow crustal anisotropy and lack of precursory changes, by Liu, Teng, Ben-Zion, Bull. Seismol. Soc. Am., 95, 354-360.

Crampin, S. and Peacock, S., 2008. A review of the current understanding of shear-wave splitting in the crust and common fallacies in interpretation. Wave Motion, 45, 675-722.

Crampin, S., Volti, T. and Stefansson, R., 1999. A successfully stress-forecast earthquake, Geophys. J. Int., 138, F1-F5.

Gamar, F. and Bernard, P., 1997. Shear wave anisotropy in the Erzincan basin and its relationship with crustal strain. J. Geophys. Res., 102, 20373-20393.

Ganas, A, Serpelloni, E., Drakatos, G., Kolligri, M., Adamis, I., Tsimi, Ch. and Batsi, E., 2009. The Mw 6.4 SW-Achaia (western Greece) earthquake of 8 June 2008: Seismological, field, GPS observations and stress modeling. J. Earthq. Eng., in press, 2009.

Gao, Y., Wang, P.-D., Zheng, S.-H., Wang, M., Chen, Y.-T. and Zhou, H.-L., 1998. Temporal changes in shear-wave splitting at an isolated swarm of small earthquakes in 1992 near Dongfang, Hainan Island, southern China. Geophys. J. Int., 135, 102-112.

Gao, Y. and Crampin, S., 2008. Shear-wave splitting and earthquake forecasting, Terra Nova, 20, 440-448.

Hao, P., Gao, Y. and Crampin, S., 2008. An Expert System for measuring shear-wave splitting above small earthquakes. Comp. Geosci., 34, 226-234.

Kaneshima, S., 1990. Origin of crustal anisotropy: Shear wave splitting studies in Japan. J. Geophys. Res., 95, 11121-11133.

Kapetanidis, V., Agalos, A., Moshou, A., Kaviris, G., Karakonstantis, A., Papadimitriou, P. and Makropoulos, K., 2008. Preliminary Results from the Study of a Seismic Swarm Occurred in February 2008 in NW Peloponnesus, Greece. Book of Abstracts (Poster), 31st Gen. Ass. ESC, p. 110, Crete, Greece.

Karnassopoulou, A., 1996. Joint investigation of source parameters and seismic anisotropy using microearthquakes in Greece, Arkansas and Northeast Brazil. PhD thesis, University of Edinburgh. 
Kaviris, G., Papadimitriou, P. and Makropoulos, K., 2008. An Overview of Anisotropy Studies in Central Greece using recordings around the Gulf of Corinth (Greece) and aftershocks of the 1999 Athens Earthquake. Short Papers, 31st ESC General Assembly, Hersonissos, Crete, Greece, 215-223.

Liu, Y., Crampin, S. and Main, I., 1997. Shear-wave anisotropy: spatial and temporal variations in time delays at Parkfield, Central California. Geophys. J. Int., 130, 771-785.

Makropoulos, K. C. and Burton, P. W., 1981 . A catalogue of seismicity in Greece and adjacent areas. Geophys. J. R. Astron. Soc., 65, 741-762.

Papadimitriou, P., Kaviris G. and Makropoulos, K., 1999. Evidence of shear-wave splitting in the Eastern Corinthian Gulf (Greece). PEPI, 114, 3-13.

Papadimitriou, P., Kaviris G., Voulgaris, N. and Kassaras, I., 2000. Shear-wave splitting analysis using aftershocks of the 7th September 1999 Earthquake: Preliminary Results. Ann. Geol. Pays Hellen., 1e Serie, T. XXXVII, FASC. B, p. 89-103. 